\title{
Weak Solutions and Energy Estimates for a Class of Nonlinear Elliptic Neumann Problems
}

\author{
Gabriele Bonanno \\ Department of Science for Engineering and Architecture (Mathematics Section) \\ Engineering Faculty, University of Messina, 98166 Messina, Italy \\ e-mail: bonanno@unime.it

\section{Giovanni Molica Bisci} \\ Dipartimento MECMAT, University of Reggio Calabria \\ Via Graziella, Feo di Vito, 89124 Reggio Calabria, Italy \\ e-mail: gmolica@unirc.it

\section{Vicențiu D. Rădulescu*} \\ Institute of Mathematics "Simion Stoilow" of the Romanian Academy \\ 014700 Bucharest, Romania \\ \& \\ Department of Mathematics, University of Craiova \\ 200585 Craiova, Romania \\ e-mail: vicentiu.radulescu@imar.ro \\ Received 05 May 2012 \\ Communicated by Norman Dancer
}

${ }^{*}$ The author acknowledges the support through Grant CNCS PCE-47/2011. 


\begin{abstract}
We establish existence results and energy estimates of solutions for a homogeneous Neumann problem involving the $p$-Laplace operator. The case of large dimensions, corresponding to the lack of compactness of $W^{1, p}(\Omega)$ in $C^{0}(\bar{\Omega})$ is also considered. In particular, for a precise localization of the parameter, the existence of a non-zero solution is established, without requiring any asymptotic condition at zero or at infinity of the nonlinear term. In the case of $(p-1)$-sublinear terms at the origin, we deduce the existence of solutions for small positive values of the parameter and we obtain that the corresponding solutions have smaller and smaller energies as the parameter goes to zero. Finally, a multiplicity result is obtained and concrete examples of applications are provided. A basic ingredient in our arguments is a recent local minimum theorem for differentiable functionals.
\end{abstract}

2010 Mathematics Subject Classification. 35J60, 47J30, 58E05.

Key words. Critical point; Variational methods; Neumann problem; sub-critical growth.

\title{
1 Introduction
}

The aim of this paper is to investigate the existence and the qualitative properties of nontrivial weak solutions of the following non-autonomous elliptic Neumann problem

$$
\begin{cases}-\Delta_{p} u+\alpha(x)|u|^{p-2} u=\lambda f(x, u) & \text { in } \Omega \\ \frac{\partial u}{\partial v}=0 & \text { on } \partial \Omega\end{cases}
$$

where $p>1$ and $\Omega \subset \mathbb{R}^{N}$ is a bounded open subset with boundary of class $C^{1}$.

As usual, $\Delta_{p}$ denotes the $p$-Laplace operator, namely $\Delta_{p} u:=\operatorname{div}\left(|\nabla u|^{p-2} \nabla u\right)$. We assume that $\alpha \in L^{\infty}(\Omega)$, with ess $\inf _{x \in \Omega} \alpha(x)>0, \lambda$ is a positive real parameter and $v$ denotes the outward unit normal to $\partial \Omega$.

Throughout this paper we assume that $f: \Omega \times \mathbb{R} \rightarrow \mathbb{R}$ is a Carathéodory function such that

$$
|f(x, t)| \leq a_{1}+a_{2}|t|^{q-1}, \quad \forall(x, t) \in \Omega \times \mathbb{R},
$$

for some non-negative constants $a_{1}, a_{2}$, where $\left.q \in\right] 1, p^{*}\left[\right.$ and $p^{*}$ stands for the critical Sobolev exponent, that is

$$
p^{*}:=\left\{\begin{array}{lll}
\frac{p N}{N-p} & \text { if } & p<N \\
+\infty & \text { if } & p \geq N .
\end{array}\right.
$$

Finally, we denote by $\mathfrak{F}_{q}$ the class of all Carathéodory functions for which condition (1.2) holds true.

The question of existence of multiple non-zero solutions has been studied for Dirichlet problems also involving the $p$-Laplace operator and there are several such papers in the literature, using different hypotheses and techniques. On the other hand, recently there have been established some multiplicity results within the Neumann setting, see the papers [3, 5, 6, 7] and [9, 25, 34]. In these works, the authors establish the existence of weak solutions for certain nonlinear elliptic problems, by imposing different kinds of oscillatory behavior on the nonlinear term.

In all the above cited contributions the basic assumption $p>N$ is imposed, which corresponds to low-dimensional problems. This dimensionality condition implies that the Sobolev space $W^{1, p}(\Omega)$ is compactly embedded in $C^{0}(\bar{\Omega})$ and this fact is used extensively in the aforementioned works; see 
Remark 3.9. In particular, in [14] and [16], the authors consider nonlinear Neumann eigenvalue problems and prove a "three solutions theorem" using an abstract multiplicity result of Ricceri [35]; see also the related papers $[19,23]$. Condition $p>N$ is also assumed in a recent work of $\mathrm{Wu}$ and Tan [36], in which there are used minimax techniques combined with critical point theory tools.

The difficulties caused by the lack of a compact embedding of $W^{1, p}(\Omega)$ in $C^{0}(\bar{\Omega})$ if $p \leq N$ are overcome by a different procedure. Indeed, to apply variational techniques to Neumann problems in the case $p \leq N$, a suitable condition on the nonlinear term $f$, which implies that the problem admits the zero solution, is usually required. Thus, three non-zero solutions can be surely obtained only when the problem is perturbed; see [16, 32] and [33].

Very recently, by using variational methods, a precise interval of values of the parameter $\lambda$, for which problem (1.1) admits at least three non-zero weak solutions has been achieved in [15, Theorem 3.1] without explicit perturbations of the nonlinear term and in higher dimension. We also recall that in the last few years, several authors have treated Neumann problems with $p$-Laplace operators (with $p \leq N$ ), by using completely different techniques. We refer to [17, 24] and the references therein; see also the works [8,20,21].

We point out that problem (1.1) arises in the study of mathematical models in biological formation theory governed by diffusion and cross-diffusion systems [28]. We refer to the recent monograph by Kristály, Rădulescu and Varga [22] for several related results and examples.

The main result of this paper (Theorem 3.1) ensures the existence of precise values of the parameter $\lambda$ for which (1.1) admits at least one non-zero weak solution, without assuming any asymptotic condition at zero or at infinity. A special case is also pointed out in Corollary 3.1. A related consequence, where the unique condition requested on the datum is the $(p-1)$-sublinearity at the origin, is also presented in this paper; see Theorem 3.2.

We also observe that, when the nonlinear term is $(p-1)$-sublinear at infinity, then the corresponding energy functional is coercive, hence the existence of one solution (possibly zero) comes from the direct methods theorem; see Remark 3.5. It is worth noticing that, in our cases, the potential may be also not coercive; see, for instance, Example 3.1. Moreover, also in presence of coercivity, our results ensure the existence of at least one non-zero weak solution.

A basic tool used in the proofs is a recent critical point theorem obtained by Bonanno in [1, Theorem 5.1] for functionals of the form $J_{\lambda}:=\Phi-\lambda \Psi$, where $\lambda$ is a positive parameter; see Theorem 2.1 below.

Consider the Sobolev space $W^{1, p}(\Omega)$ endowed with the norm

$$
\|u\|:=\left(\int_{\Omega}|\nabla u(x)|^{p} d x+\int_{\Omega} \alpha(x)|u(x)|^{p} d x\right)^{1 / p},
$$

which is equivalent to the usual one.

We state here, as an example, the following special case of our results; see also Remark 3.8.

Theorem 1.1 Let $p>1$ and $f: \mathbb{R} \rightarrow \mathbb{R}$ be a continuous function such that

$$
\lim _{t \rightarrow 0^{+}} \frac{f(t)}{t^{p-1}}=+\infty, \quad \text { and } \quad \lim _{|t| \rightarrow \infty} \frac{f(t)}{|t|^{s}}=0,
$$

for some $0 \leq s<p^{*}-1$. Then, there exists $\lambda^{\star}>0$ such that, for every $\left.\lambda \in\right] 0, \lambda^{\star}[$, the following autonomous Neumann problem

$$
\begin{cases}-\Delta_{p} u+\alpha(x)|u|^{p-2} u=\lambda f(u) & \text { in } \Omega \\ \partial u / \partial v=0 & \text { on } \partial \Omega\end{cases}
$$


admits at least one non-zero weak solution $u_{0, \lambda} \in W^{1, p}(\Omega)$. Moreover, we have $\left\|u_{0, \lambda}\right\| \rightarrow 0$ as $\lambda \rightarrow 0^{+}$ and the mapping

$$
\lambda \mapsto \frac{1}{p}\left(\int_{\Omega}\left|\nabla u_{0, \lambda}(x)\right|^{p} d x+\int_{\Omega} \alpha(x)\left|u_{0, \lambda}(x)\right|^{p} d x\right)-\lambda \int_{\Omega}\left(\int_{0}^{u_{0, \lambda}(x)} f(x, t) d t\right) d x,
$$

is negative and strictly decreasing in $] 0, \lambda^{\star}[$.

We observe that Theorem 1.1 ensures the existence of one non-zero weak solution even if $f(0)=$ 0 provided that (1.3) holds, and, if $\alpha$ is not constant, the solution is not constant. Moreover, a concrete expression for $\lambda^{\star}$, that appears in the above result, is given in Remark 3.4. Furthermore, we just point out that in Corollary 3.2, adding to hypotheses of Theorem 3.2 the classical Ambrosetti and Rabinowitz condition, namely (AR), a second weak solution is achieved; see Example 3.2. It is worth noticing that in Corollary 3.2 the assumptions are different from those usually required when applying the mountain pass theorem; see Remark 3.10 .

The paper is organized as follows. In Section 2, we recall some basic definitions and our main tool, while Section 3 is devoted to our main results and examples.

\section{Preliminaries}

Let $W^{1, p}(\Omega)$ endowed with the norm $\|\cdot\|$. From standard variational arguments, the weak solutions of (1.1) are the critical points of the $C^{1}$-functional given by

$$
J_{\lambda}(u):=\frac{1}{p}\left(\int_{\Omega}|\nabla u(x)|^{p} d x+\int_{\Omega} \alpha(x)|u(x)|^{p} d x\right)-\lambda \int_{\Omega}\left(\int_{0}^{u(x)} f(x, t) d t\right) d x,
$$

for every $u \in W^{1, p}(\Omega)$.

A function $u: \Omega \rightarrow \mathbb{R}$ is said to be a weak solution of problem (1.1) if $u \in W^{1, p}(\Omega)$ and

$$
\int_{\Omega}|\nabla u(x)|^{p-2} \nabla u(x) \cdot \nabla v(x) d x+\int_{\Omega} \alpha(x)|u(x)|^{p-2} u(x) v(x) d x=\lambda \int_{\Omega} f(x, u(x)) v(x) d x,
$$

for every $v \in W^{1, p}(\Omega)$.

Let $X$ be a real Banach space. We say that a continuously Gâteaux differentiable functional $J: X \rightarrow \mathbb{R}$ verifies the Palais-Smale condition (in short (PS)-condition) if any sequence $\left\{u_{n}\right\}$ such that

$\left(\mathrm{j}_{1}\right)\left\{J\left(u_{n}\right)\right\}$ is bounded,

$\left(\mathrm{j}_{2}\right) \lim _{n \rightarrow \infty}\left\|J^{\prime}\left(u_{n}\right)\right\|_{X^{*}}=0$,

has a convergent subsequence.

For an exhaustive treatment of these topics we refer to [27, 30] and the references therein.

Let $\Phi, \Psi: X \rightarrow \mathbb{R}$ be two continuously Gâteaux differentiable functions. Set

$$
J=\Phi-\Psi
$$

and fix $r_{1}, r_{2} \in[-\infty,+\infty]$, with $r_{1}<r_{2}$; we say that function $J$ verifies the Palais-Smale condition cut off lower at $r_{1}$ and upper at $r_{2}$ (in short ${ }^{\left[r_{1}\right]}(\mathrm{PS})^{\left[r_{2}\right]}$-condition) if any sequence $\left\{u_{n}\right\}$ such that $\left(\mathrm{j}_{1}\right)$, $\left(\mathrm{j}_{2}\right)$ hold and 


$$
\left(\mathrm{j}_{3}\right) r_{1}<\Phi\left(u_{n}\right)<r_{2}, \quad \forall n \in \mathbb{N},
$$

has a convergent subsequence.

Clearly, if $r_{1}=-\infty$ and $r_{2}=+\infty$ it coincides with the classical (PS)-condition. Moreover, if $r_{1}=-\infty$ and $r_{2} \in \mathbb{R}$ it is denoted by (PS ${ }^{\left[r_{2}\right]}$, while if $r_{1} \in \mathbb{R}$ and $r_{2}=+\infty$ it is denoted by ${ }^{\left[r_{1}\right]}(\mathrm{PS})$. Furthermore, if $J$ satisfies ${ }^{\left[r_{1}\right]}(\mathrm{PS})^{\left[r_{2}\right]}$-condition, then it satisfies ${ }^{\left[\rho_{1}\right]}(\mathrm{PS})^{\left[\rho_{2}\right]}$-condition for all $\rho_{1}, \rho_{2} \in[-\infty,+\infty]$ such that $r_{1} \leq \rho_{1}<\rho_{2} \leq r_{2}$.

In particular, we deduce that if $J$ satisfies the classical (PS)-condition, then it satisfies ${ }^{\left[\rho_{1}\right]}(\mathrm{PS})^{\left[\rho_{2}\right]_{-}}$ condition for all $\rho_{1}, \rho_{2} \in[-\infty,+\infty]$ with $\rho_{1}<\rho_{2}$. Set

$$
\beta\left(r_{1}, r_{2}\right):=\inf _{v \in \Phi^{-1}(] r_{1}, r_{2}[)} \frac{\sup _{u \in \Phi^{-1}(] r_{1}, r_{2}[)} \Psi(u)-\Psi(v)}{r_{2}-\Phi(v)},
$$

and

$$
\rho_{2}\left(r_{1}, r_{2}\right):=\sup _{v \in \Phi^{-1}(] r_{1}, r_{2}[)} \frac{\Psi(v)-\sup _{\left.\left.u \in \Phi^{-1}(]-\infty, r_{1}\right]\right)} \Psi(u)}{\Phi(v)-r_{1}},
$$

for all $r_{1}, r_{2} \in \mathbb{R}$, with $r_{1}<r_{2}$.

A crucial role in the existence proof of one non-zero weak solution for problem (1.1) is played by the following version of an abstract local minimum theorem obtained in [1, Theorem 5.1], which we recall here for convenience.

Theorem 2.1 Let $X$ be a real Banach space and let $\Phi, \Psi: X \rightarrow \mathbb{R}$ be two continuously Gâteaux differentiable functions. Assume that there are $r_{1}, r_{2} \in \mathbb{R}$, with $r_{1}<r_{2}$, such that

$$
\beta\left(r_{1}, r_{2}\right)<\rho_{2}\left(r_{1}, r_{2}\right)
$$

where $\beta$ and $\rho_{2}$ are given by (2.5) and (2.6), and for each

$$
\lambda \in] \frac{1}{\rho_{2}\left(r_{1}, r_{2}\right)}, \frac{1}{\beta\left(r_{1}, r_{2}\right)}[,
$$

the function $J_{\lambda}:=\Phi-\lambda \Psi$ satisfies ${ }^{\left[r_{1}\right]}(\mathrm{PS})^{\left[r_{2}\right]}$-condition. Then, for all $\left.\lambda \in\right] \frac{1}{\rho_{2}\left(r_{1}, r_{2}\right)}, \frac{1}{\beta\left(r_{1}, r_{2}\right)}[$ there is $u_{0, \lambda} \in \Phi^{-1}(] r_{1}, r_{2}[)$ such that $J_{\lambda}\left(u_{0, \lambda}\right) \leq J_{\lambda}(u)$ for all $u \in \Phi^{-1}(] r_{1}, r_{2}[)$ and $J_{\lambda}^{\prime}\left(u_{0, \lambda}\right)=0$.

Remark 2.1 Theorem 2.1 has been inspired from the Ricceri variational principle; see [31, Theorem 2.5]. With respect to the mentioned principle, the above result furnishes a more precise localization of the minimum $u_{0, \lambda}$, and, in particular, since $\Phi\left(u_{0, \lambda}\right)>r_{1}$, in many applications we have $u_{0, \lambda} \neq 0$ as, for instance, in Theorem 3.1. Moreover, we also emphasize that in Theorem 2.1 no weak lower semicontinuity assumption is requested on the contrary of [31, Theorem 2.5].

\section{Main results}

Recall that $p^{*}$ stands for the critical exponent of the Sobolev embedding $W^{1, p}(\Omega) \hookrightarrow L^{q}(\Omega)$. Thus, if $p<N$ then $p^{*}=N p /(N-p)$ and for every $q \in\left[1, p^{*}\right]$ there exists a positive constant $\kappa_{q}$ such that

$$
\|u\|_{L^{q}(\Omega)} \leq \kappa_{q}\|u\|
$$

for every $u \in W^{1, p}(\Omega)$. When $p \geq N$ this inequality holds for any $q \in[1,+\infty[$. 
Moreover, for every two nonnegative constants $\gamma, \delta$, with $\gamma \neq \delta$, we set

$$
a_{\gamma}(\delta):=\frac{A(\gamma)-q \int_{\Omega} F(x, \delta) d x}{\|\alpha\|_{L^{1}(\Omega)}\left(\gamma^{p}-\delta^{p}\right) q},
$$

where

$$
A(\gamma):=q\|\alpha\|_{L^{1}(\Omega)}^{1 / p} \gamma \kappa_{1} a_{1}+\|\alpha\|_{L^{1}(\Omega)}^{q / p} \gamma^{q} \kappa_{q}^{q} a_{2}
$$

and

$$
F(x, \xi):=\int_{0}^{\xi} f(x, t) d t
$$

for every $(x, \xi) \in \Omega \times \mathbb{R}$.

The following result establishes a qualitative property of solutions to problem (1.1).

Theorem 3.1 Let $f \in \mathfrak{F}_{q}$ and assume that there exist three real constants $\gamma_{1}, \gamma_{2}$ and $\delta$, with $0 \leq$ $\gamma_{1}<\delta<\gamma_{2}$, such that

$$
a_{\gamma_{2}}(\delta)<a_{\gamma_{1}}(\delta)
$$

Then, for each parameter $\lambda$ belonging to

$$
] \frac{1}{p a_{\gamma_{1}}(\delta)}, \frac{1}{p a_{\gamma_{2}}(\delta)}[
$$

problem (1.1) admits at least one non-zero weak solution $u_{0, \lambda} \in W^{1, p}(\Omega)$, such that

$$
\|\alpha\|_{L^{1}(\Omega)}^{1 / p} \gamma_{1}<\left\|u_{0, \lambda}\right\|<\|\alpha\|_{L^{1}(\Omega)}^{1 / p} \gamma_{2} .
$$

Proof. Our aim is to apply Theorem 2.1. Hence, let $X:=W^{1, p}(\Omega)$ and consider the functionals $\Phi, \Psi: X \rightarrow \mathbb{R}$ defined by

$$
\Phi(u):=\frac{\|u\|^{p}}{p}, \quad \Psi(u):=\int_{\Omega} F(x, u(x)) d x, \quad \forall u \in X .
$$

Clearly, $\Phi: X \rightarrow \mathbb{R}$ is a coercive and continuously Gâteaux differentiable functional. On the other hand, $\Psi$ is well-defined and continuously Gâteaux differentiable. More precisely, we have

$$
\begin{gathered}
\Phi^{\prime}(u)(v)=\int_{\Omega}|\nabla u(x)|^{p-2} \nabla u(x) \cdot \nabla v(x) d x+\int_{\Omega} \alpha(x)|u(x)|^{p-2} u(x) v(x) d x, \\
\Psi^{\prime}(u)(v)=\int_{\Omega} f(x, u(x)) v(x) d x,
\end{gathered}
$$

for every $u, v \in X$.

Fix $\lambda>0$. A critical point of the functional $J_{\lambda}:=\Phi-\lambda \Psi$ is a function $u \in X$ such that

$$
\Phi^{\prime}(u)(v)-\lambda \Psi^{\prime}(u)(v)=0,
$$

for every $v \in X$. Hence, the critical points of the functional $J_{\lambda}$ are weak solutions of problem (1.1). At this point, let us observe that $\Phi\left(0_{X}\right)=\Psi\left(0_{X}\right)=0$. Moreover, since $f \in \mathfrak{F}$, we have

$$
F(x, \xi) \leq a_{1}|\xi|+a_{2} \frac{|\xi|^{q}}{q},
$$


for every $(x, \xi) \in \Omega \times \mathbb{R}$. Now, taking into account relation (3.11), it follows that

$$
\Psi(u)=\int_{\Omega} F(x, u(x)) d x \leq a_{1}\|u\|_{L^{1}(\Omega)}+\frac{a_{2}}{q}\|u\|_{L^{q}(\Omega)}^{q} .
$$

Then, for every $u \in X$ such that $\Phi(u) \leq r$, owing to (3.7), we obtain

$$
\Psi(u) \leq(p r)^{1 / p} \kappa_{1} a_{1}+\frac{p^{q / p} \kappa_{q}^{q} a_{2}}{q} r^{q / p} .
$$

Therefore

$$
\sup _{\left.\left.u \in \Phi^{-1}(]-\infty, r\right]\right)} \Psi(u) \leq(p r)^{1 / p} \kappa_{1} a_{1}+\frac{p^{q / p} \kappa_{q}^{q} a_{2}}{q} r^{q / p}
$$

Next, we set

$$
r_{1}:=\frac{\|\alpha\|_{L^{1}(\Omega)}}{p} \gamma_{1}^{p}, \quad r_{2}:=\frac{\|\alpha\|_{L^{1}(\Omega)}}{p} \gamma_{2}^{p}, \quad \text { and } \quad u_{\delta}(x):=\delta, \quad \text { for every } x \in \Omega .
$$

Then $u_{\delta} \in X$ and

$$
\Phi\left(u_{\delta}\right)=\frac{1}{p}\left(\int_{\Omega}\left|\nabla u_{\delta}(x)\right|^{p} d x+\int_{\Omega} \alpha(x)\left|u_{\delta}(x)\right|^{p} d x\right)=\frac{\delta^{p}}{p}\|\alpha\|_{L^{1}(\Omega)} .
$$

Taking into account that $\gamma_{1}<\delta<\gamma_{2}$, by a direct computation, one has $r_{1}<\Phi\left(u_{\delta}\right)<r_{2}$. Moreover,

$$
\Psi\left(u_{\delta}\right)=\int_{\Omega} F\left(x, u_{\delta}(x)\right) d x=\int_{\Omega} F(x, \delta) d x .
$$

From (3.12) it follows that

$$
\sup _{u \in \Phi^{-1}(]-\infty, r_{2}[)} \Psi(u) \leq\left(p r_{2}\right)^{1 / p} \kappa_{1} a_{1}+\frac{p^{q / p} \kappa_{q}^{q} a_{2}}{q} r_{2}^{q / p} .
$$

and

$$
\sup _{\left.\left.u \in \Phi^{-1}(]-\infty, r_{1}\right]\right)} \Psi(u) \leq\left(p r_{1}\right)^{1 / p} \kappa_{1} a_{1}+\frac{p^{q / p} \kappa_{q}^{q} a_{2}}{q} r_{1}^{q / p} .
$$

We have $r_{1}<\Phi\left(u_{\delta}\right)<r_{2}$. On the other hand,

$$
\beta\left(r_{1}, r_{2}\right):=\inf _{v \in \Phi^{-1}(] r_{1}, r_{2}[)} \frac{\sup _{u \in \Phi^{-1}(] r_{1}, r_{2}[)} \Psi(u)-\Psi(v)}{r_{2}-\Phi(v)} \leq \frac{\sup _{u \in \Phi^{-1}(]-\infty, r_{2}[)} \Psi(u)-\Psi\left(u_{\delta}\right)}{r_{2}-\Phi\left(u_{\delta}\right)}
$$

and

$$
\rho_{2}\left(r_{1}, r_{2}\right):=\sup _{v \in \Phi^{-1}(] r_{1}, r_{2}[)} \frac{\Psi(v)-\sup _{\left.\left.u \in \Phi^{-1}(]-\infty, r_{1}\right]\right)} \Psi(u)}{\Phi(v)-r_{1}} \geq \frac{\Psi\left(u_{\delta}\right)-\sup _{\left.u \in \Phi^{-1}\left(1-\infty, r_{1}\right]\right)} \Psi(u)}{\Phi\left(u_{\delta}\right)-r_{1}} .
$$

Hence, by using the notation (3.8), from (3.14) and (3.15) together with (3.16), it follows that

$$
\beta\left(r_{1}, r_{2}\right) \leq p a_{\gamma_{2}}(\delta), \quad \text { and } \quad \rho_{2}\left(r_{1}, r_{2}\right) \geq p a_{\gamma_{1}}(\delta) .
$$


Finally, hypothesis (3.10) yields

$$
\beta\left(r_{1}, r_{2}\right)<\rho_{2}\left(r_{1}, r_{2}\right) .
$$

Now, from [1, Proposition 2.1], the functional $J_{\lambda}$ satisfies ${ }^{\left[r_{1}\right]}(\mathrm{PS})^{\left[r_{2}\right]}$-condition for all $r_{1}$ and $r_{2}$ with $r_{1}<r_{2}<+\infty$. Therefore, owing to Theorem 2.1, for each $\left.\lambda \in\right] \frac{1}{p a_{\gamma_{1}}(\delta)}, \frac{1}{p a_{\gamma_{2}}(\delta)}\left[\right.$, the functional $J_{\lambda}$ admits at least one critical point $u_{0, \lambda}$ such that

$$
r_{1}<\Phi\left(u_{0, \lambda}\right)<r_{2}
$$

that is

$$
\|\alpha\|_{L^{1}(\Omega)}^{1 / p} \gamma_{1}<\left\|u_{0, \lambda}\right\|<\|\alpha\|_{L^{1}(\Omega)}^{1 / p} \gamma_{2} .
$$

This completes the proof.

Remark 3.1 If $p<N$, a concrete upper bound for the constants $\kappa_{q}$ in Theorem 3.1 (hence for the values of constant $A$ defined as in (3.9)) can be obtained considering an open and convex set $\Omega \subset \mathbb{R}^{N}$ of diameter $d$ and Lebesgue measure "meas $(\Omega)$ ". In this case, if $q \in\left[1, p^{*}[\right.$, we have

$$
\kappa_{q} \leq S_{q} \frac{(1+N) \operatorname{meas}(\Omega)^{\frac{p-q}{p q}}}{\min \{1, \underset{x \in \Omega}{\operatorname{essinf}} \alpha(x)\}^{1 / p}}
$$

where

$$
S_{q}:=\left\{\begin{array}{l}
\max \left\{1, \frac{d}{N}\right\} \quad \text { if } \quad q \in[1, p[, \\
\max \left\{1, \frac{d}{N}\left(\frac{p q-N(q-p)}{p q+(p-q)}\right)^{\frac{q-p(1+q)}{p q}}\right\} \quad \text { if } \quad q \in\left[p, p^{*}[,\right.
\end{array}\right.
$$

see, for instance, Remark 3.2 in [15] and, more generally, the exhaustive book [13]. Moreover, we point out that the following alternative holds: either $q \in\left[p, p^{*}[\right.$ and the diameter of $\Omega$ is sufficiently small, that is,

$$
d<N\left(\frac{p q-N(q-p)}{p q+(p-q)}\right)^{\frac{p(1+q)-q}{p q}}
$$

or $q \in[1, p[$ and $d<N$. Inequality (3.17) can be rewritten as

$$
\kappa_{q} \leq \frac{(1+N) \operatorname{meas}(\Omega)^{\frac{p-q}{p q}}}{\min \left\{1, \operatorname{essinf}_{x \in \Omega} \alpha(x)\right\}^{1 / p}}
$$

as observed in [15, Remark 3.3].

On the other hand, in the low-dimensional case that corresponds to $p>N$, assuming $\Omega$ to be convex and by using [5, Remark 2.1], an easy computation ensures that for every $q \geq 1$, we have

$$
\kappa_{q} \leq 2^{\frac{p-1}{p}} \max \left\{\frac{1}{\|\alpha\|_{L^{1}(\Omega)}^{\frac{1}{p}}}, \frac{d}{N^{\frac{1}{p}}}\left(\frac{p-1}{p-N} \operatorname{meas}(\Omega)\right)^{\frac{p-1}{p}} \frac{\|\alpha\|_{\infty}}{\|\alpha\|_{L^{1}(\Omega)}}\right\} \operatorname{meas}(\Omega)^{1 / q} .
$$


Remark 3.2 If $p>N$ and $\Omega$ is convex, by using again [5, Remark 2.1], a concrete estimate of the solution $u_{0, \lambda}$ in $C^{0}(\bar{\Omega})$ can be achieved. Precisely, we have

$$
\left\|u_{0, \lambda}\right\|_{\infty} \leq 2^{\frac{p-1}{p}} \max \left\{\frac{1}{\|\alpha\|_{L^{1}(\Omega)}^{\frac{1}{p}}}, \frac{d}{N^{\frac{1}{p}}}\left(\frac{p-1}{p-N} \operatorname{meas}(\Omega)\right)^{\frac{p-1}{p}} \frac{\|\alpha\|_{\infty}}{\|\alpha\|_{L^{1}(\Omega)}}\right\}\|\alpha\|_{L^{1}(\Omega)}^{1 / p} \gamma_{2} .
$$

Now, we point out a particular case of Theorem 3.1.

Corollary 3.1 Let $f \in \mathfrak{F}_{q}$ and assume that there exist two positive constants $\gamma$ and $\delta$, with $\gamma>\delta$, such that

$$
\frac{\int_{\Omega} F(x, \delta) d x}{\delta^{p}}>\frac{A(\gamma)}{q \gamma^{p}} .
$$

Then, for each parameter $\lambda$ belonging to

$$
\int \frac{\|\alpha\|_{L^{1}(\Omega)} \delta^{p}}{p \int_{\Omega} F(x, \delta) d x}, \frac{\|\alpha\|_{L^{1}(\Omega)} \gamma^{p} q}{p A(\gamma)}[,
$$

problem (1.1) admits at least one non-zero weak solution $u_{0, \lambda} \in W^{1, p}(\Omega)$, such that

$$
\left\|u_{0, \lambda}\right\|<\|\alpha\|_{L^{1}(\Omega)}^{1 / p} \gamma .
$$

Proof. Our aim is to apply Theorem 3.1. To this end we pick $\gamma_{1}=0$ and $\gamma_{2}:=\gamma$. Bearing in mind (3.8), we obtain

$$
a_{\gamma}(\delta)=\frac{A(\gamma)-q \int_{\Omega} F(x, \delta) d x}{\|\alpha\|_{L^{1}(\Omega)}\left(\gamma^{p}-\delta^{p}\right) q},
$$

as well as

$$
a_{0}(\delta)=\frac{\int_{\Omega} F(x, \delta) d x}{\delta^{p}\|\alpha\|_{L^{1}(\Omega)}} .
$$

Now, inequality (3.20) immediately yields

$$
a_{\gamma}(\delta)<a_{0}(\delta)
$$

Hence, Theorem 3.1 ensures the conclusion. A direct consequence of Corollary 3.1 is the following property.

Theorem 3.2 Let $f \in \mathfrak{F}_{q}$ and assume that

$$
\lim _{\xi \rightarrow 0^{+}} \frac{\int_{\Omega} F(x, \xi) d x}{\xi^{p}}=+\infty .
$$

Furthermore, let $\gamma>0$ and set

$$
\lambda_{\gamma}^{\star}:=\frac{q\|\alpha\|_{L^{1}(\Omega)}}{p} \frac{\gamma^{p}}{A(\gamma)} .
$$


Then, for every $\lambda \in] 0, \lambda_{\gamma}^{\star}\left[\right.$, the problem (1.1) admits at least one non-zero weak solution $u_{0, \lambda} \in$ $W^{1, p}(\Omega)$ such that $\left\|u_{0, \lambda}\right\|<\|\alpha\|_{L^{1}(\Omega)}^{1 / p} \gamma$ and

$$
\lim _{\lambda \rightarrow 0^{+}}\left\|u_{0, \lambda}\right\|=0
$$

Proof. Fix $\lambda \in] 0, \lambda_{\gamma}^{\star}[$. From (3.21) there exists a positive constant $\delta$ with $\delta<\gamma$ such that

$$
\frac{\|\alpha\|_{L^{1}(\Omega)} \delta^{p}}{p \int_{\Omega} F(x, \delta) d x}<\lambda<\frac{\|\alpha\|_{L^{1}(\Omega)} \gamma^{p} q}{A(\gamma) p}
$$

Hence, owing to Corollary 3.1, problem (1.1) admits at least one non-zero weak solution $u_{0, \lambda}$, such that $\left\|u_{0, \lambda}\right\|<\|\alpha\|_{L^{1}(\Omega)}^{1 / p} \gamma$. Then, for every $\left.\lambda \in\right] 0, \lambda_{\gamma}^{\star}[$, there exists at least one non-zero weak solution $u_{0, \lambda} \in \Phi^{-1}(] 0, r_{2}[)$ of problem (1.1) and one has

$$
\left\|u_{0, \lambda}\right\|<\|\alpha\|_{L^{1}(\Omega)}^{1 / p} \gamma .
$$

for every $\lambda \in] 0, \lambda_{\gamma}^{\star}[$. Therefore, from (1.2), taking into account (3.7) and (3.22), it follows that

$$
\left|\int_{\Omega} f\left(x, u_{0, \lambda}(x)\right) u_{0, \lambda}(x) d x\right| \leq\|\alpha\|_{L^{1}(\Omega)}^{1 / p} \gamma \kappa_{1} a_{1}+\|\alpha\|_{L^{1}(\Omega)}^{q / p} \gamma^{q} \kappa_{q}^{q} a_{2},
$$

for every $\lambda \in] 0, \lambda_{\gamma}^{\star}\left[\right.$. Now, $J_{\lambda}^{\prime}\left(u_{0, \lambda}\right)=0$, for every $\left.\lambda \in\right] 0, \lambda_{\gamma}^{\star}\left[\right.$ and in particular $J_{\lambda}^{\prime}\left(u_{0, \lambda}\right)\left(u_{0, \lambda}\right)=0$, that is,

$$
\left\|u_{0, \lambda}\right\|^{p}=\lambda \int_{\Omega} f\left(x, u_{0, \lambda}(x)\right) u_{0, \lambda}(x) d x,
$$

for every $\lambda \in] 0, \lambda_{\gamma}^{\star}[$. Then, from (3.23), it follows that

$$
\lim _{\lambda \rightarrow 0^{+}}\left\|u_{0, \lambda}\right\|^{p}=\lim _{\lambda \rightarrow 0^{+}} \lambda \Psi^{\prime}\left(u_{0, \lambda}\right)\left(u_{0, \lambda}\right)=0,
$$

that implies $\lim _{\lambda \rightarrow 0^{+}}\left\|u_{0, \lambda}\right\|=0$. The proof is complete.

Remark 3.3 We claim that under the above assumptions, the mapping $\lambda \mapsto J_{\lambda}\left(u_{0, \lambda}\right)$ is negative and strictly decreasing in $] 0, \lambda_{\gamma}^{\star}\left[\right.$. Indeed, the restriction of the functional $J_{\lambda}$ to $\Phi^{-1}(] 0, r_{2}[)$, where $r_{2}:=\left(\|\alpha\|_{L^{1}(\Omega)} / p\right) \gamma_{2}^{p}$, admits a global minimum, which is a critical point (local minimum) of $J_{\lambda}$ in $X$. Moreover, since $u_{\delta}:=\delta \in \Phi^{-1}(] 0, r_{2}[)$ and

$$
\frac{\Phi\left(u_{\delta}\right)}{\Psi\left(u_{\delta}\right)}=\frac{\|\alpha\|_{L^{1}(\Omega)} \delta^{p}}{p \int_{\Omega} F(x, \delta) d x}<\lambda
$$

we have

$$
J_{\lambda}\left(u_{0, \lambda}\right) \leq J_{\lambda}\left(u_{\delta}\right)=\Phi\left(u_{\delta}\right)-\lambda \Psi\left(u_{\delta}\right)<0 .
$$

Next, we observe that

$$
J_{\lambda}(u)=\lambda\left(\frac{\Phi(u)}{\lambda}-\Psi(u)\right),
$$

for every $u \in X$ and fix $0<\lambda_{1}<\lambda_{2}<\lambda_{\gamma}^{\star}$. Set

$$
m_{\lambda_{1}}:=\left(\frac{\Phi\left(u_{0, \lambda_{1}}\right)}{\lambda_{1}}-\Psi\left(u_{0, \lambda_{1}}\right)\right)=\inf _{u \in \Phi^{-1}(] 0, r_{2}[)}\left(\frac{\Phi(u)}{\lambda_{1}}-\Psi(u)\right),
$$


and

$$
m_{\lambda_{2}}:=\left(\frac{\Phi\left(u_{0, \lambda_{2}}\right)}{\lambda_{2}}-\Psi\left(u_{0, \lambda_{2}}\right)\right)=\inf _{u \in \Phi^{-1}(] 0, r_{2}[)}\left(\frac{\Phi(u)}{\lambda_{2}}-\Psi(u)\right) .
$$

Clearly, as claimed before, $m_{\lambda_{i}}<0$ (for $i=1$,2), and $m_{\lambda_{2}} \leq m_{\lambda_{1}}$ thanks to $\lambda_{1}<\lambda_{2}$. Then the mapping $\lambda \mapsto J_{\lambda}\left(u_{0, \lambda}\right)$ is strictly decreasing in $] 0, \lambda_{\gamma}^{\star}[$ owing to

$$
J_{\lambda_{2}}\left(u_{0, \lambda_{2}}\right)=\lambda_{2} m_{\lambda_{2}} \leq \lambda_{2} m_{\lambda_{1}}<\lambda_{1} m_{\lambda_{1}}=J_{\lambda_{1}}\left(u_{0, \lambda_{1}}\right) .
$$

This concludes the proof of our claim.

Remark 3.4 Roughly speaking, Theorem 3.2 ensures that if $f$ has the global growth given by (1.2) and the asymptotic condition at zero (3.21) is verified then, for every parameter $\lambda$ belonging to the real interval $\left.\Lambda_{\Omega}:=\right] 0, \lambda^{\star}[$, where

$$
\lambda^{\star}:=\frac{q\|\alpha\|_{L^{1}(\Omega)}}{p} \sup _{\gamma>0} \frac{\gamma^{p}}{A(\gamma)}
$$

the problem (1.1) admits at least one non-zero solution $u_{0, \lambda} \in W^{1, p}(\Omega)$. Moreover $\left\|u_{0, \lambda}\right\| \rightarrow 0$, as $\lambda \rightarrow 0^{+}$. Furthermore, from a direct computation, it follows that

$$
\lambda^{\star}=\left\{\begin{array}{l}
+\infty \quad \text { if } 1<q<p \\
\frac{1}{\kappa_{p}^{p} a_{2}} \quad \text { if } q=p \\
\left.\frac{q\|\alpha\|_{L^{1}(\Omega)}^{(p-1) / p} \bar{\gamma}_{\max }^{p-1}}{p\left(q \kappa_{1} a_{1}+\|\alpha\|_{L^{1}(\Omega)}^{(q-1) / p} \kappa_{q}^{q} a_{2} \bar{\gamma}_{\max }^{q-1}\right)} \quad \text { if } q \in\right] p, p^{*}[
\end{array}\right.
$$

where

$$
\bar{\gamma}_{\max }:=\frac{1}{\|\alpha\|_{L^{1}(\Omega)}^{1 / p}}\left(q \frac{\kappa_{1} a_{1}}{\kappa_{q}^{q} a_{2}}\left(\frac{p-1}{q-p}\right)\right)^{1 /(q-1)} .
$$

We also note that, in the case $q \in] p, p^{*}\left[\right.$, one has $\left\|u_{0, \lambda}\right\|<\|\alpha\|_{L^{1}(\Omega)}^{1 / p} \bar{\gamma}_{\max }$, uniformly for every $\lambda \in \Lambda_{\Omega}$.

Remark 3.5 We emphasize that, in particular, if $f$ is $(p-1)$-sublinear at infinity, Theorem 3.2 ensures that, problem (1.1) admits at least one non-zero weak solution for each positive parameter $\lambda$. It is worth noticing that, in our case, the attained solution, as affirmed, is non-zero, while the classical direct method approach, that can be adopted in this setting, ensures the existence of at least one solution that may be zero.

Remark 3.6 For completeness we observe that if $f$ is a non-negative function our results guarantee that the attained weak solution is non-negative. To this end, let $u_{0}$ be a weak solution of problem (1.1). Arguing by contradiction, assume that the set $A:=\left\{x \in \Omega: u_{0}(x)<0\right\}$ has positive Lebesgue measure. Put $\bar{v}(x)=\min \left\{0, u_{0}(x)\right\}$ for all $x \in \Omega$. Clearly, $\bar{v} \in W^{1, p}(\Omega)$ and

$$
\begin{gathered}
\int_{\Omega}\left|\nabla u_{0}(x)\right|^{p-2} \nabla u_{0}(x) \cdot \nabla \bar{v}(x) d x+\int_{\Omega} \alpha(x)\left|u_{0}(x)\right|^{p-2} u_{0}(x) \bar{v}(x) d x \\
-\lambda \int_{\Omega} f\left(x, u_{0}(x)\right) \bar{v}(x) d x=0,
\end{gathered}
$$


that is,

$$
\int_{A}\left|\nabla u_{0}(x)\right|^{p} d x+\int_{A} \alpha(x)\left|u_{0}(x)\right|^{p} d x=\lambda \int_{A} f\left(x, u_{0}(x)\right) u_{0}(x) d x \leq 0 .
$$

Hence

$$
\int_{A}\left|\nabla u_{0}(x)\right|^{p} d x+\int_{A} \alpha(x)\left|u_{0}(x)\right|^{p} d x=0 .
$$

Then, $u_{0}=0$ almost everywhere in $A$. This is not possible by the definition of $A$, so it follows that $u_{0}$ is non-negative.

Remark 3.7 A careful analysis of the proof of Theorem 3.2 ensures that the result still remains true after replacing condition (3.21) with the more general assumption

$$
\limsup _{\xi \rightarrow 0^{+}} \frac{\int_{\Omega} F(x, \xi) d x}{\xi^{p}}=+\infty .
$$

Moreover, in the autonomous case, the previous asymptotic condition at zero assume the form

$$
\limsup _{\xi \rightarrow 0^{+}} \frac{F(\xi)}{\xi^{p}}=+\infty
$$

Remark 3.8 We just observe that Theorem 1.1 in Introduction is a simple consequence of Theorem 3.2 and Remark 3.3. Indeed, if

$$
\lim _{t \rightarrow 0^{+}} \frac{f(t)}{t^{p-1}}=+\infty
$$

holds, then hypothesis (3.26) is automatically verified. Moreover, hypothesis

$$
\lim _{|t| \rightarrow \infty} \frac{f(t)}{|t|^{s}}=0
$$

where $0 \leq s<p^{*}-1$, ensures that $f$ has a subcritical growth.

In the sequel, there is an example of application of our results.

Example 3.1 Let $\Omega$ be a bounded open subset of $\mathbb{R}^{N}$ with $1<p<N$ and consider the following problem

$$
\begin{cases}-\Delta_{p} u+|u|^{p-2} u=\lambda\left[\eta(x)|u|^{r-2} u+\beta(x)|u|^{s-2} u\right] & \text { in } \Omega \\ \frac{\partial u}{\partial v}=0 & \text { on } \partial \Omega,\end{cases}
$$

where $\eta, \beta: \Omega \rightarrow \mathbb{R}$ are two continuous positive and bounded functions, $1<r<p$ and $p<s<p^{*}$. Then, for every

$$
\lambda \in] 0, \frac{s \operatorname{meas}(\Omega)^{(p-1) / p} \bar{\gamma}_{\max }^{p-1}}{2 p \max \left\{\|\eta\|_{\infty},\|\beta\|_{\infty}\right\}\left(s \kappa_{1}+\operatorname{meas}(\Omega)^{(s-1) / p} \kappa_{s}^{s} \bar{\gamma}_{\max }^{s-1}\right)}[,
$$

where

$$
\bar{\gamma}_{\max }:=\frac{1}{\operatorname{meas}(\Omega)^{1 / p}}\left(s \frac{\kappa_{1}}{\kappa_{s}^{s}}\left(\frac{p-1}{s-p}\right)\right)^{1 /(s-1)}
$$

the problem (3.28) admits at least one non-zero weak solution $u_{0, \lambda} \in W^{1, p}(\Omega)$ such that

$$
\left\|u_{0, \lambda}\right\|<\left(s \frac{\kappa_{1}}{\kappa_{s}^{s}}\left(\frac{p-1}{s-p}\right)\right)^{1 /(s-1)}
$$


and $\lim _{\lambda \rightarrow 0^{+}}\left\|u_{0, \lambda}\right\|=0$. To prove this, we can apply Theorem 3.2 with

$$
f(x, t):=\eta(x)|t|^{r-2} t+\beta(x)|t|^{s-2} t,
$$

for every $(x, t) \in \Omega \times \mathbb{R}$. Indeed, it is easy to verify that $f \in \mathfrak{F}_{s}$ since

$$
|f(x, t)| \leq 2 \max \left\{\|\eta\|_{\infty},\|\beta\|_{\infty}\right\}\left(1+|t|^{s-1}\right), \quad \forall(x, t) \in \Omega \times \mathbb{R} .
$$

Moreover, a direct computation shows that

$$
\lim _{\xi \rightarrow 0^{+}} \frac{\int_{\Omega} F(x, \xi) d x}{\xi^{p}} \geq \frac{\operatorname{meas}(\Omega) \inf _{x \in \Omega} \eta(x)}{r} \lim _{\xi \rightarrow 0^{+}} \frac{1}{\xi^{p-r}}=+\infty .
$$

Hence, all the assumptions of Theorem 3.2 are verified and the conclusion follows.

Remark 3.9 A similar version of Theorem 3.2 in the low-dimensional case has been studied in [10] and [11]. In their setting, taking advantage of the compact embedding of $W^{1, p}(\Omega)$ into $C^{0}(\bar{\Omega})$, condition (1.2) can be removed and the authors are able to treat also cases with exponential growth; see, for instance, [10, Example 3.1]. Furthermore, a direct comparison with Theorems 1 and 2 of [37] can be find in [10, Remark 3.6]. In conclusion, taking into account Remarks 3.6 and 3.7, we emphasize that, Theorem 3.2 represents an extension of [10, Theorem 3.3] and [11, Theorem 4.1] to the case $p \leq N$. Moreover, in the low-dimensional case, the additional presence of the growth assumption (1.2) may determine, in some case, an improvement of the intervals of parameters for which our approach guarantee the existence of at least one non-zero weak solution. Indeed, let $p>N$ and assume $f: \Omega \times \mathbb{R} \rightarrow \mathbb{R}$ to be $L^{1}$-Carathéodory. As [11, Theorem 3.4] shows, the existence of at least one non-zero weak solution to Neumann problem is obtained for every $\lambda \in] 0, \lambda_{f}[$, where

$$
\lambda_{f}:=\frac{1}{p c^{p}} \sup _{\gamma>0} \frac{\gamma^{p}}{\int_{\Omega} \max _{|\xi| \leq \gamma} F(x, \xi) d x},
$$

and

$$
c:=\sup _{u \in W^{1, p}(\Omega) \backslash\{0\}} \frac{\sup _{x \in \bar{\Omega}}|u(x)|}{\left(\int_{\Omega}|\nabla u(x)|^{p} d x+\int_{\Omega} \alpha(x)|u(x)|^{p} d x\right)^{1 / p}}<+\infty .
$$

If the function $f$ satisfies also the growth condition (1.2) the necessity of to compare the intervals ] $0, \lambda^{\star}[$ and $] 0, \lambda_{f}$ [ naturally arises. We exhibit a concrete example in which $] 0, \lambda_{f}[\subset] 0, \lambda^{\star}[$. For our goal, let us take a positive constant $d$ and set $\Omega=] 0,2 d$. At this point, consider the ordinary non-autonomous Neumann problem

$$
\left\{\begin{array}{l}
\left.-u^{\prime \prime}+\frac{k u}{2}=\lambda \beta(x)\left(1+|u|^{q-1}\right) \quad \text { in } \quad\right] 0,2 d[ \\
u^{\prime}(0)=u^{\prime}(2 d)=0,
\end{array}\right.
$$

where $\beta:[0,2 d] \rightarrow \mathbb{R}$ is a positive and continuous function, $q>2$ and

$$
0<k<\frac{\|\beta\|_{L^{1}(0,2 d)}}{2\|\beta\|_{\infty}(c d)^{2}}
$$

Set

$$
\mathcal{N}(c, q):=\frac{1}{2 c^{2}}\left(\frac{q-2}{q-1}\right)\left(\frac{q}{q-2}\right)^{1 /(q-1)}
$$


and

$$
G(\beta, q):=\left(\frac{d}{2\|\beta\|_{L^{1}(0,2 d)}}\right)\left(\frac{q-2}{q-1}\right)\left(\frac{q}{q-2}\right)^{1 /(q-1)} .
$$

Hence, bearing in mind the above results, problem (3.29) admits a non-zero classical solution for every $\lambda \in] 0, \lambda_{f}$ [ as well as for every $\left.\lambda \in\right] 0, \lambda^{\star}\left[\right.$. Now, taking into account that $1 / c^{2} \leq k d$, simple computations yield

$$
\lambda_{f}=\frac{q}{2 c^{2}\|\beta\|_{L^{1}(0,2 d)}} \sup _{\gamma>0}\left(\frac{\gamma}{q+\gamma^{q-1}}\right) \leq k\left(\frac{q d}{2\|\beta\|_{L^{1}(0,2 d)}}\right) \sup _{\gamma>0}\left(\frac{\gamma}{q+\gamma^{q-1}}\right) .
$$

Then, since

$$
\sup _{\gamma>0}\left(\frac{\gamma}{q+\gamma^{q-1}}\right)=\frac{1}{q}\left(\frac{q-2}{q-1}\right)\left(\frac{q}{q-2}\right)^{1 /(q-1)},
$$

one has

$$
\lambda_{f} \leq k\left(\frac{d}{2\|\beta\|_{L^{1}}}\right)\left(\frac{q-2}{q-1}\right)\left(\frac{q}{q-2}\right)^{1 /(q-1)}=k G(\beta, q) .
$$

On the other hand, thanks to $\kappa_{s} \leq(2 d)^{1 / s} c$, for every $s \geq 1$, it follows that

$$
\lambda^{\star}=\frac{q}{\|\beta\|_{\infty}} \sup _{\gamma>0}\left(\frac{\gamma}{q \sqrt{2} \kappa_{1}+2^{q / 2} \kappa_{q}^{q} \gamma^{q-1}}\right) \geq \frac{q}{2 d\|\beta\|_{\infty}} \sup _{\gamma>0}\left(\frac{\gamma}{q \sqrt{2} c+2^{q / 2} c^{q} \gamma^{q-1}}\right) .
$$

Hence, since

$$
\sup _{\gamma>0}\left(\frac{\gamma}{q \sqrt{2} c+2^{q / 2} c^{q} \gamma^{q-1}}\right)=\frac{1}{2 c^{2} q}\left(\frac{q-2}{q-1}\right)\left(\frac{q}{q-2}\right)^{1 /(q-1)}
$$

one has

$$
\lambda^{\star} \geq\left(\frac{1}{2 d\|\beta\|_{\infty}}\right) \mathcal{N}(c, q) .
$$

Then, inequalities (3.31) and (3.32) together with condition (3.30) yield

$$
\lambda_{f} \leq k G(\beta, q)<\left(\frac{1}{2 d\|\beta\|_{\infty}}\right) \mathcal{N}(c, q) \leq \lambda^{\star} .
$$

In conclusion, the expected strict inclusion $] 0, \lambda_{f}[\subset] 0, \lambda^{\star}[$ holds.

In the sequel we prove how the previous results can be employed in order to pass from the existence of at least one nontrivial solution to the existence of at lest two nontrivial solutions. This goal will be achieved making use of the particular nature of the first solution found, namely it is a local minimum. This information will be used to assure the existence of a second solution as a critical point of mountain pass type. In this direction, we begin with the following theorem, where the celebrated Ambrosetti-Rabinowitz condition is required. As usual, this assumption plays a crucial role in proving that every Palais-Smale sequence is bounded, as well as that the so called 'mountain pass geometry' is satisfied. 
Corollary 3.2 Let $f: \mathbb{R} \rightarrow \mathbb{R}$ be a continuous function such that

$$
|f(t)| \leq a_{1}+a_{2}|t|^{q-1}, \quad \forall t \in \mathbb{R},
$$

for some non-negative constants $a_{1}, a_{2}$, where $\left.q \in\right] p, p^{*}$ [. Furthermore, assume that condition (3.26) holds in addition to

(AR) there are constants $\mu>p$ and $r>0$ such that, for all $|\xi| \geq r$, one has

$$
0<\mu F(\xi) \leq \xi f(\xi)
$$

Then, for each $\lambda \in \Lambda_{\Omega}$, problem (1.1) admits at least two weak solutions. If, in addition to the above assumptions, one has $f(0) \neq 0$, the attained solutions are non-zero.

Proof. Fix $\lambda \in \Lambda_{\Omega}$. Owing to (3.33) and (3.26), Theorem 3.2 ensures that the problem (1.1) admits at least one weak non-zero solution $u_{1}$ which is a local minimum of the functional $J_{\lambda}$ as defined in the proof of Theorem 3.1. Now, we can assume that $u_{1}$ is a strict local minimum for $J_{\lambda}$ in $X$. Therefore, there is $\rho>0$ such that

$$
\inf _{\left\|u-u_{1}\right\|=\rho} J_{\lambda}(u)>J_{\lambda}\left(u_{1}\right)
$$

Furthermore, from (AR)-condition, by standard computations, one has that $J_{\lambda}$ is unbounded from below. So, there is $u_{2}$ such that $J_{\lambda}\left(u_{2}\right)<J_{\lambda}\left(u_{1}\right)$, for which $J_{\lambda}$ satisfies mountain pass geometry. At this point, again exploiting (AR), it follows that the functional $J_{\lambda}$ satisfies the (PS)-condition. Hence, the celebrated Ambrosetti-Rabinowitz theorem ensures the existence of a critical point $\tilde{u}$ of $J_{\lambda}$ such that $J_{\lambda}(\tilde{u})>J_{\lambda}\left(u_{1}\right)$. So, $u_{1}$ and $\tilde{u}$ are two distinct weak solutions of (1.1) and the proof is complete.

Example 3.2 Let $\Omega$ be a bounded open subset of $\mathbb{R}^{N}$ with $1<p<N$ and consider the following problem

$$
\begin{cases}-\Delta_{p} u+\alpha(x)|u|^{p-2} u=\lambda\left(1+u^{2 m-1}\right) & \text { in } \Omega \\ \frac{\partial u}{\partial v}=0 & \text { on } \partial \Omega\end{cases}
$$

where $m$ is a positive integer such that $1<p<2 m<p^{*}$. Then, for every

$$
\lambda \in] 0, \frac{2 m-p}{p \kappa_{1}(2 m-1)}\left(\frac{2 m \kappa_{1}(p-1)}{\kappa_{2 m}^{2 m}(2 m-p)}\right)^{\frac{p-1}{2 m-1}}[,
$$

problem (3.34) admits at least two non-zero $(f(0) \neq 0)$ weak solutions. To prove this, we can apply Corollary 3.2 with

$$
f(t):=1+t^{2 m-1}
$$

for every $t \in \mathbb{R}$. Indeed, clearly the function $f$ satisfies (3.33) and, since

$$
\lim _{t \rightarrow 0^{+}} \frac{f(t)}{t^{p-1}}=+\infty,
$$

also condition (3.26) holds true. Moreover, taking into account that

$$
\lim _{|\xi| \rightarrow \infty} \frac{\xi f(\xi)}{F(\xi)}=2 m \lim _{|\xi| \rightarrow \infty} \frac{\xi^{2 m}+\xi}{\xi^{2 m}+2 m \xi}=2 m>p,
$$

there exist $\mu>p$ and $r>0$ such that

$$
0<\mu F(\xi) \leq \xi f(\xi),
$$

for every $|\xi|>r$. Hence, all the assumptions of Corollary 3.2 are verified and the conclusion follows. 
Remark 3.10 Applying the mountain pass theorem to nonlinear differential problems, involving the Laplace operator $-\Delta u$, it is usual to require that $\lim _{t \rightarrow 0^{+}} \frac{f(t)}{t}=0$; see, for instance, condition $\left(p_{3}\right)$ in [30, Theorem 2.15]. On the contrary, in Example 3.2, the above assumption at zero is not verified. We also note that, very recently, Neumann problems have been studied without (AR)-condition by using a Cerami-type condition; see [18] and references therein, as well as Remark 3.11 below. Even in this case, hypothesis $\lim _{t \rightarrow 0^{+}} \frac{f(t)}{t}<+\infty$ is requested. Hence, [18, Theorem 3.6] cannot be applied to problem (3.34) in Example 3.2.

Remark 3.11 The (AR)-condition, adopted in Corollary 3.2, implies that the energy functional $J_{\lambda}$ is unbounded from below and satisfies the classical (PS)-condition, so that the classical mountain pass theorem can be applied. Therefore, instead of assumption (AR), a Cerami-type condition could be exploited in Corollary 3.2 in order to obtain the second solution. We refer, for instance, to [4, 18, 29] for more details on related topics.

\section{References}

[1] G. Bonanno, A critical point theorem via the Ekeland variational principle, Nonlinear Anal. 75 (2012), 2992-3007.

[2] G. Bonanno and P. Candito, Three solutions to a Neumann problem for elliptic equations involving the p-Laplacian, Arch. Math. (Basel) 80 (2003), 424-429.

[3] G. Bonanno and G. D'Aguì, Multiplicity results for a perturbed elliptic Neumann problem, Abstr. Appl. Anal. 2010 (2010), 1-10.

[4] G. Bonanno and R. Livrea, Existence and multiplicity of periodic solutions for second order Hamiltonian systems depending on a parameter, preprint.

[5] G. Bonanno and G. Molica Bisci, A remark on a perturbed Neumann problem, Stud. Univ. Babes-Bolyai Math. LV 4 (2010), 17-25.

[6] G. Bonanno, G. Molica Bisci, and V. Rădulescu, Existence of three solutions for a non-homogeneous Neumann problem through Orlicz-Sobolev spaces, Nonlinear Anal. 74 (2011), 4785-4795.

[7] G. Bonanno, G. Molica Bisci, and V. Rădulescu, Infinitely many solutions for a class of nonlinear eigenvalue problems in Orlicz-Sobolev spaces, C.R. Acad. Sci. Paris, Ser. I 349 (2011), 263-268.

[8] G. Bonanno, G. Molica Bisci, and V. Rădulescu, Multiple solutions of generalized Yamabe equations on Riemannian manifolds and applications to Emden-Fowler problems, Nonlinear Anal. Real World Appl. 12 (2011), 2656-2665.

[9] G. Bonanno, D. Motreanu, and P. Winkert, Variational-hemivariational inequalities with small perturbations of nonhomogeneous Neumann boundary conditions, J. Math. Anal. Appl. 381 (2011), 627-637.

[10] G. Bonanno and P.F. Pizzimenti, Neumann boundary value problems with non-coercive potential, Mediterr. J. Math. 9 (2012), 601-609.

[11] G. Bonanno and A. Sciammetta, Existence and multiplicity results to Neumann problems for elliptic equations involving the p-Laplacian, J. Math. Anal. Appl. 390 (2012), 59-67.

[12] H. Brézis, Functional Analysis, Sobolev Spaces and Partial Differential Equations, Universitext, Springer, New York, 2011.

[13] V.I. Burenkov, Sobolev Spaces on Domains, Teubner-Texte zur Mathematik [Teubner Texts in Mathematics], 137. B. G. Teubner Verlagsgesellschaft mbH, Stuttgart, 1998.

[14] F. Cammaroto, A. Chinnì, and B. Di Bella, Some multiplicity results for quasilinear Neumann problems, Archiv der Math. 86 (2006), 154-162. 
[15] G. D'Aguì and G. Molica Bisci, Three non-zero solutions for elliptic Neumann problems, Anal. Appl. 9 (2011), 383-394.

[16] F. Faraci, Multiplicity results for a Neumann problem involving the p-Laplacian, J. Math. Anal. Appl. 277 (2003), 180-188.

[17] L. Gasiński and N.S. Papageorgiou, Nontrivial solutions for a class of resonant p-Laplacian Neumann problems, Nonlinear Anal. 71 (2009), 6365-6372.

[18] L. Gasiński and N.S. Papageorgiou, Nontrivial solutions for Neumann problems with an indefinite linear part, Appl. Math. Comput. 217 (2010), 2666-2675.

[19] A. Kristály, M. Mihailescu, and V. Rădulescu, Two nontrivial solutions for a non-homogeneous Neumann problem: an Orlicz-Sobolev space setting, Proc. Roy. Soc. Edinburgh Sect. A 139 (2009), 367-379.

[20] A. Kristály and N.S. Papageorgiou, Multiple nontrivial solutions for Neumann problems involving the p-Laplacian: a Morse theoretical approach, Adv. Nonlinear Stud. 10 (2010), 83-107.

[21] A. Kristály, N.S. Papageorgiou, and Cs. Varga, Multiple solutions for a class of Neumann elliptic problems on compact Riemannian manifolds with boundary, Canad. Math. Bull. 53 (2010), 674-683.

[22] A. Kristály, V. Rădulescu, and Cs. Varga, Variational Principles in Mathematical Physics, Geometry, and Economics: Qualitative Analysis of Nonlinear Equations and Unilateral Problems, Encyclopedia of Mathematics and its Applications, No. 136, Cambridge University Press, Cambridge, 2010.

[23] A. Kristály and D. Repovš, Multiple solutions for a Neumann system involving subquadratic nonlinearities, Nonlinear Anal. 74 (2011), 2127-2132.

[24] S.A. Marano and G. Molica Bisci, Multiplicity results for a Neumann problem with p-Laplacian and non-smooth potential, Rend. Circ. Mat. Palermo 55 (2006), 113-122.

[25] S.A. Marano and D. Motreanu, Infinitely many critical points of nondifferentiable functions and applications to a Neumann-type problem involving the p-Laplacian, J. Differential Equations 182 (2002), $108-120$.

[26] S.A. Marano and N.S. Papageorgiou, On a Neumann problem with p-Laplacian and non-smooth potential, Differential Integral Equations 19 (2006), 1301-1320.

[27] D. Motreanu and V. Rădulescu, Variational and Non-variational Methods in Nonlinear Analysis and Boundary Value Problems, Nonconvex Optimization and Applications, Kluwer Academic Publishers, 2003.

[28] W.M. Ni, Diffusion, cross-diffusion and their spike layer states, Notices Amer. Math. Soc. 45 (1998), 9-18.

[29] N.S. Papageorgiou and E.M. Rocha, Pairs of positive solutions for p-Laplacian equations with sublinear and superlinear nonlinearities which do not satisfy the AR-condition, Nonlinear Anal. 70 (2009), 38543863.

[30] P.H. Rabinowitz, Minimax Methods in Critical Point Theory with Applications to Differential Equations, CBMS Reg. Conferences in Math., vol. 65, Amer. Math. Soc. Providence, RI, 1985.

[31] B. Ricceri, A general variational principle and some of its applications, J. Comput. Appl. Math. 113 (2000), 401-410.

[32] B. Ricceri, A multiplicity theorem for the Neumann problem, Proc. Amer. Math. Soc. 134 (2006), 11171124.

[33] B. Ricceri, A note on the Neumann problem, Complex Var. Elliptic Equ. 55 (2010), 593-599.

[34] B. Ricceri, Infinitely many solutions of the Neumann problem for elliptic equations involving the pLaplacian, Bull. London Math. Soc. 33 (2001), 331-340.

[35] B. Ricceri, On a three critical points theorem, Archiv der Math. 75 (2000), 220-226.

[36] X. Wu and K.K. Tan, On the existence and multiplicty of solutions of Neumann boundary problems for quasilinear elliptic equations, Nonlinear Anal. 65 (2006), 1334-1347.

[37] L. Zhilong, Existence of positive solutions of superlinear second-order Neumann boundary value problem, Nonlinear Anal. 72 (2010), 3216-3221. 